\title{
Variante y reescritura en Anabella y El pentágono de Antonio Di Benedetto: dos obras, un mismo proceso escritural
}

\author{
Variant and rewriting in Anabella and El pentágono by Antonio Di \\ Benedetto: two works, the same scriptural process
}

Mateo Green

Universidad Nacional de Córdoba

\begin{abstract}
Resumen: El presente trabajo tiene como principal objetivo comparar, con herramientas conceptuales de la Crítica Genética, dos ediciones de una obra de Antonio Di Benedetto y registrar las variantes existentes entre una y otra para constatar las operaciones de reescritura introducidas por el autor, entendiendo ambas versiones como materiales de un proceso escritural en la cronología de la génesis de una obra. Posteriormente, a partir del registro de estas variantes, se intentarán establecer algunos planteos críticos en relación a la reescritura del texto. Fundamentalmente, se busca demostrar que en la segunda edición que compone este proceso escritural no solo se hicieron correcciones menores, sino que el texto fue sometido a una relectura, en el modo que indica Grésillon (2008: 169), tras un largo período entre redacciones que llevó a una reescritura y a una modificación sustancial de algunos de sus sentidos.
\end{abstract}

Palabras clave: Di Benedetto, reescritura, proceso escritural.

Abstract: The main objective of the present paper is to compare, with conceptual tools of Genetic Criticism, two editions of a work by Antonio Di Benedetto, in order to register the existing variants between one and the other as to verify the rewriting operations introduced by the author, understanding both versions as materials of a scriptural process in the chronology of the genesis of a text. Subsequently, based on the registration of these variants, we aim to establish some critical points in relation to the rewriting. Fundamentally, we seek to show that in the second edition that makes up this scriptural process, not only minor corrections were made, but the text was subjected to rereading, as indicated by Grésillon (2008: 169), after a long period between redactions which led to a rewriting of the text and a substantial modification of some of its meanings.

Keywords: Di Benedetto, rewriting, scriptural process.

Recibido: 08/11/2020

Aceptado: 06/02/2021 


\section{ANTONIO DI BENEDETTO, ESCRITOR}

Antonio Di Benedetto, nacido en 1922 en la ciudad de Mendoza y, por tanto, en la periferia cultural de la Argentina de la época, excesivamente centralizada en Buenos Aires. Destacado periodista, hizo su carrera en el diario Los Andes además de otros medios, actividad que eventualmente le valió el injusto encarcelamiento por la dictadura militar de 1976 y el posterior exilio. Escritor.

¿Cómo hacer una semblanza biográfica de aquellos que, como decía Foucault, «escriben para perder el rostro» (2008: 29)? La vida de Di Benedetto es una vida susceptible de ser pensada desde su relación con la escritura. Interpretado por la crítica como escritor antirregionalista, experimental, atípico, objetivista, fantástico; como novelista histórico, costumbrista y existencialista; clasificaciones por demás diversas que evidencian la dificultad de abordaje de su obra. Una obra que, para Premat, antes que un objeto definido presenta un problema de lectura (2017: 11).

Publicó, fuera de su actividad periodística, cinco novelas: El pentágono (1955, reeditada como Anabella en 1974), Zama (1956), El silenciero (1964), Los Suicidas (1969) y Sombras, nada más (1985). Además de seis libros de cuentos: Mundo Animal (1953), Grot (1957, reeditado como Cuentos Claros en 1969), Declinación y Ángel (1958), El Cariño de los tontos (1961), Absurdos (1978) y Cuentos del exilio (1983). Premiado y traducido a varios idiomas en vida, adaptado al cine recientemente ${ }^{1}$, Di Benedetto fue condenado a un breve olvido en la década que siguió a su muerte. Gracias a los esfuerzos de su amigo el escritor Juan José Saer ${ }^{2}$ y a un renovado interés de público y la crítica, la totalidad de su obra ha sido publicada en los últimos años por la editorial Adriana Hidalgo, incluyendo sus Escritos periodísticos (1943-1986).

Escritor prolífico a quien las vicisitudes personales sometieron a un agotamiento progresivo (Premat, 2017: 11). De la totalidad de su obra, seis libros se publicarían en nueve años — de 1954 a 1961—, sus beginnings para Premat; y los restantes seis en el lapso de veinticinco años que van desde 1961 hasta su fallecimiento en 1986. Es de la escritura en esos beginnings y de su relación con la etapa inmediatamente posterior de lo que nos ocuparemos aquí.

\section{OBRA, EDICIÓN Y OTRA VEZ OBRA}

En 1955 la editorial doble p publica El pentágono. Novela en forma de cuentos, el segundo libro de Antonio Di Benedetto y el primero editado en Buenos Aires. Escrita en la década del 40 y motivada por cierto afán rupturista respecto de la novela tradicional,

\footnotetext{
${ }^{1}$ Los suicidas de Juan Villegas en 2006 y la aclamada Zama de Lucrecia Martel en 2017.

${ }^{2}$ Sobre todo, en dos textos aparecidos en El concepto de ficción (1997), Zama y Antonio Di Benedetto, y un prólogo a El silenciero de 1999.
} 
representa, debido a su estructura singular, una tentativa del autor por «contar de otra manera» (Di Benedetto, 1974: 12). Este modo diferente de contar, no definido por Di Benedetto, es reducido por Premat a sus características esenciales:

...fragmentación, paradójica artificialidad (ya que pretende representar las peculiaridades subjetivas de una experiencia), extrañamiento lingüístico, técnicas paracinematográficas, inmediatez, tendencia al minimalismo y a la abstracción, concentración alrededor de una conciencia reflectora y en general narradora. (Premat, 2004: 299)

Si bien, a causa de estos procedimientos, la crítica en un principio asoció éste y otros textos iniciales de Di Benedetto al Nouveau roman —o Nueva novela—, y al Objetivismo propuestos por Alain Robbe Grillet y Nathalie Sarraute, esto ha sido debidamente puesto en discusión por Filer (1982), Néspolo (2004) y Premat (2004 y 2017), además de que el mismo Di Benedetto dijo en una entrevista de 1963 con el diario La Nación que él no tenía «la menor idea de que en Francia o en cualquier parte estuviese en marcha o estaba por ponerse en marcha un movimiento de esas características» (Filer, 1982:20). También hay que tener en cuenta que el término Nouveau Roman no sería acuñado hasta 1957, dos años después de la publicación de El pentágono y que, si bien los dos cuentos que componen Declinación y Ángel, comúnmente asociados a la obra de Sarraute — por equívoco, según Filer ${ }^{3}$-, fueron publicados en 1958, su escritura data de comienzos de esa década (Filer, 1982:20), cuando esa influencia no era posible en la Argentina. Sí eran posibles, sin embargo, otras influencias probadas en Di Benedetto, casualmente cercanas a las que fundamentan la Nueva novela: Kafka, Joyce y Borges (Premat, 2017:12). La escritura experimental y anticipatoria de los beginnings de Di Benedetto, novedosa en la narrativa argentina de entonces ${ }^{4}$, debe entenderse fuera de su posible asociación con el movimiento francés, a pesar de que luego Di Benedetto haya entrado en contacto con la obra de Sarraute o Robbe Grillet y, a partir de allí, releído la suya propia en miras a la revisión de El pentágono.

La primera edición de 1955 de El pentágono, a pesar de otorgarle cierta presencia nacional a Di Benedetto, carece de notoriedad por el pequeño volumen de la tirada (Varela, 2004: 29).

\footnotetext{
${ }^{3}$ Filer sostiene la primacía de la influencia del cine en Di Benedetto como explicación a los procedimientos cercanos al objetivismo. A su vez, la compleja relación entre el autor y el cine es desarrollada extensamente por Néspolo en la segunda parte de Ejercicios de pudor: «La sugestión del cine».

${ }^{4}$ Néspolo (2004) ha trabajado en profundidad la cuestión de la novedad que Di Benedetto supone en la narrativa argentina de mitad de S. XX, pero para mayor brevedad y focalización en los primeros textos, véase el artículo de Premat: Así se nace, Vanguardia, estilo, extrañeza en Di Benedetto (2017).
} 

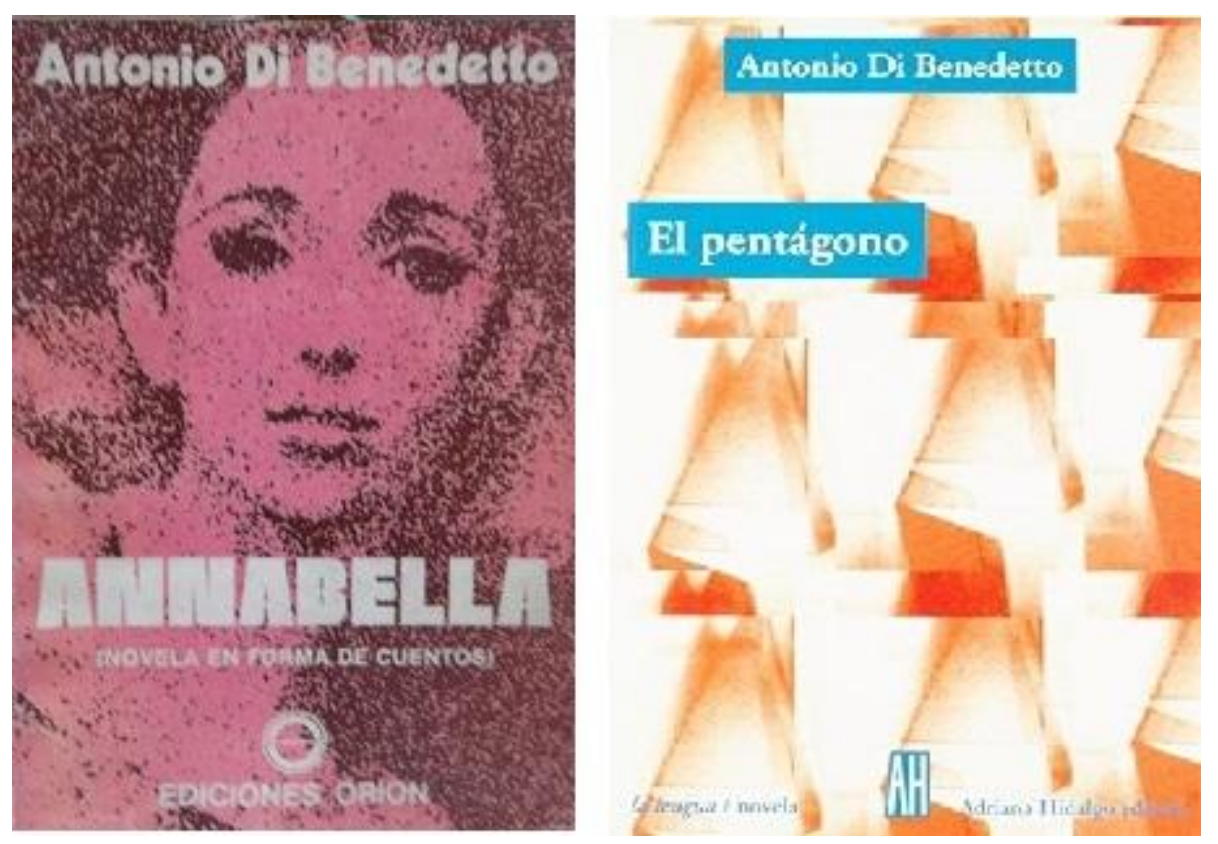

Los relatos que componen esta «novela en forma de cuentos» narran el clásico tema del triángulo amoroso desde el punto de vista de un narrador en primera persona que cuenta, por un lado y de manera metaficcional, el triángulo entre él, Laura, un amor ideal que solo existe en sus fantasías, y Rolando Fortuna; y por el otro el existente entre él, su esposa Bárbara y el amante de ésta, Orlando Sabino. Estos dos triángulos paralelos, narrados de manera fragmentaria en diferentes cuentos y teniendo por vértice al narrador protagonista, configuran en su superposición, un pentágono, figura que da nombre a la primera edición de la novela:
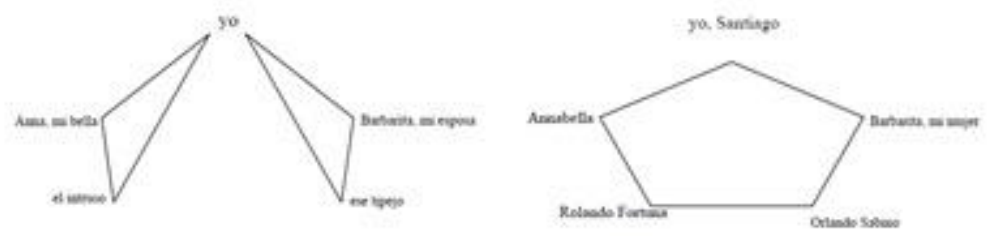

(Di Benedetto, 2018a: 30-31)

En 1974, casi veinte años después de esta poco conocida publicación de El pentágono, Ediciones Orión vuelve a editar el texto bajo el nombre de Anabella. (Novela en forma de cuentos), con la aclaración: «El Pentágono, pasada en limpio por el autor» (Di Benedetto, 1974: 5). Esta nueva edición no solo supone una recontextualización de la obra «después del éxito de la Novela objetiva y del boom de la literatura hispanoamericana» (Varela: 129), sino también una verdadera reescritura, asociada a una posibilidad de relectura luego del 
mencionado éxito del Objetivismo y la Nueva novela que el propio autor referencia en un apartado incluido en esta edición denominado «Indicios» (Di Benedetto, 1974: 9). A pesar de que en ese apartado Di Benedetto afirma que «Sobre las correcciones para la edición ha de considerarse, simplemente, que he tomado mi escrito y lo he pasado en limpio» (1974: 13), la cantidad y densidad de las variaciones, empezando por el cambio del título que se corresponde a la sustitución del nombre del personaje femenino más importante - Laura por Anabella-, nos permiten afirmar que no se trata de meras correcciones, sino de variaciones insertas en un proceso escritural que hace de Anabella una reescritura de El pentágono, llevada a cabo por un escritor más maduro que, a la fecha, ya había publicado sus novelas más importantes: Zama (1956), El silenciero (1964) y Los suicidas (1969).

Élida Lois afirma que «El objeto de análisis de la crítica genética son los documentos escritos [...] que, agrupados en conjuntos coherentes, constituyen la huella visible de un proceso creativo» (2005a: 56). Es sobre esta base teórica que entendemos las dos ediciones mencionadas como el conjunto de documentos sobre el cual es posible dar cuenta de una serie de variaciones, un proceso de reescritura, y establecer algunos lineamientos críticos relativos a dicho proceso. Para tal fin utilizaremos una reedición integra de El pentágono, llevada a cabo por Adriana Hidalgo Editora en 2018, fiel al texto de 1955, y la Primera Edición de Anabella de 1974 hecha por Ediciones Orión.

\section{VARIANTES Y REESCRITURA}

Para Lois, el resultado de las indagaciones geneticistas no es el texto como conjunto acabado «sino el proceso mismo. Así, la investigación no se traduce en la mera inversión de una dinámica con el objeto de rescatar la 'palabra más auténtica', se trata de enfocar un nuevo objeto de análisis: la escritura in progress» (2005a: 51). Es por ello que consideramos nuestros materiales, las ediciones de El pentágono y Anabella, objeto de análisis de la crítica genética en tanto «documentos escritos [...] que, agrupado en conjuntos coherentes, constituyen la huella visible de un proceso creativo» (2005a: 56). Para los cambios entre una edición y la otra, atendemos al concepto de «variante», que el glosario de Grésillon define como:

... unidad verbal que es diferente de otra forma, anterior o posterior; distintas versiones de un texto se distinguen por sus variantes; la noción de variante supone, en principio, una versión considerada como punto de referencia. (2008: 296)

Las variantes deben ser entendidas como parte de un proceso de reescritura, constituido por una serie de operaciones específicas que es necesario registrar:

En los estudios genéticos, la "reescritura" se define como una operación escritural que vuelve sobre lo ya escrito (ya se trate de palabras, frases, párrafos, capítulos o textos enteros) para 
sustituirlos o suprimirlos. Así, aunque la reescritura se ofrece como una combinatoria de operaciones múltiples y heterogéneas [...], la sustitución (el reemplazo de un elemento A por un elemento B) y la supresión constituyen sus operaciones básicas. (Lois, 2005b: 133)

Aparte de la sustitución y la supresión, consideraremos también las adiciones en tanto las define Grésillon como «unidad añadida» (2008: 168).

Registraremos entonces las variantes más importantes encontradas en una lectura atenta de las dos versiones, excluyendo por motivos de extensión y objetivos las que consideramos de índole gramatical, sintáctica u ortográfica, es decir, las meras correcciones. Para su registro tendremos en cuenta:

a) Variantes globales y de estructura: aquellas que afectan a la totalidad del texto o a la estructura del mismo como cambios en nombres de personajes (repetidos siempre de manera idéntica), en títulos de partes o capítulos, orden de capítulos o partes, etc.

b) Variantes de reescritura: aquellas alteraciones que constituyan una nueva escritura del texto base por adición, supresión o sustitución léxica, fraseológica o de un pasaje, y que puedan identificarse como huellas en un proceso escritural, es decir, que introduzcan cambios de sentido en el texto más allá de la mera corrección (variantes mínimas que no modifican sustancialmente el sentido, sintácticas u ortográficas), registradas según su orden de aparición. Ver al respecto, concepto de «variante libre» en el glosario de Grésillon (2005: 296).

\section{VARIANTES GLOBALES Y DE ESTRUCTURA}

\begin{tabular}{|c|c|c|}
\hline & $\begin{array}{l}\text { Reedición de Adriana Hidalgo } 2018 \\
\text { (fiel al texto de 1955) }\end{array}$ & $\begin{array}{l}\text { Edición de } 1974 \text { por Ediciones } \\
\text { Orion }\end{array}$ \\
\hline Título de la obra & $\begin{array}{l}\text { El pentágono } \\
\text { Novela en forma de cuentos }\end{array}$ & $\begin{array}{l}\text { Anabella } \\
\text { (Novela en forma de cuentos) }\end{array}$ \\
\hline Paratextos & & $\begin{array}{l}\text { - Se introduce, debajo de la página } \\
6 \text { donde se indica el título, una } \\
\text { aclaración que reza «El } \\
\text { Pentágono, pasado en limpio por } \\
\text { el autor» } \\
\text { - Se introduce en la página } 8 \text { una } \\
\text { dedicatoria: } \\
\text { «A mi madre, Sara. } \\
\text { A mi hermana, Carmen Aída» } \\
\end{array}$ \\
\hline $\begin{array}{l}\text { Nombres } \\
\text { personajes }\end{array}$ & $\begin{array}{l}\text { - «Laura», la integrante del primer } \\
\text { triángulo amoroso, amor ideal del } \\
\text { protagonista narrador. } \\
\text { - «Laurita», la hija que el protagonista } \\
\text { tiene con Laura en el relato Primer }\end{array}$ & $\begin{array}{l}\text { - El nombre de «Laura» cambia en } \\
\text { todos los relatos del texto a } \\
\text { «Anabella». No hay ninguna } \\
\text { mención al nombre Laura más } \\
\text { que en «Indicios», el apartado }\end{array}$ \\
\hline
\end{tabular}




\begin{tabular}{|c|c|c|}
\hline & $\begin{array}{l}\text { desalojo, año 1788; segundo } \\
\text { desalojo, año } 1951\end{array}$ & $\begin{array}{l}\text { incluido como aclaraciones del } \\
\text { autor a esta edición. } \\
\text { La hija del protagonista con } \\
\text { Anabella en el relato El } \\
\text { desalojado manso pasa a } \\
\text { llamarse, consecuentemente al } \\
\text { cambio del nombre de la madre, } \\
\text { Anibella. }\end{array}$ \\
\hline $\begin{array}{l}\text { Estructura } \\
\text { general: partes, } \\
\text { distribución de } \\
\text { relatos y sus títulos }\end{array}$ & 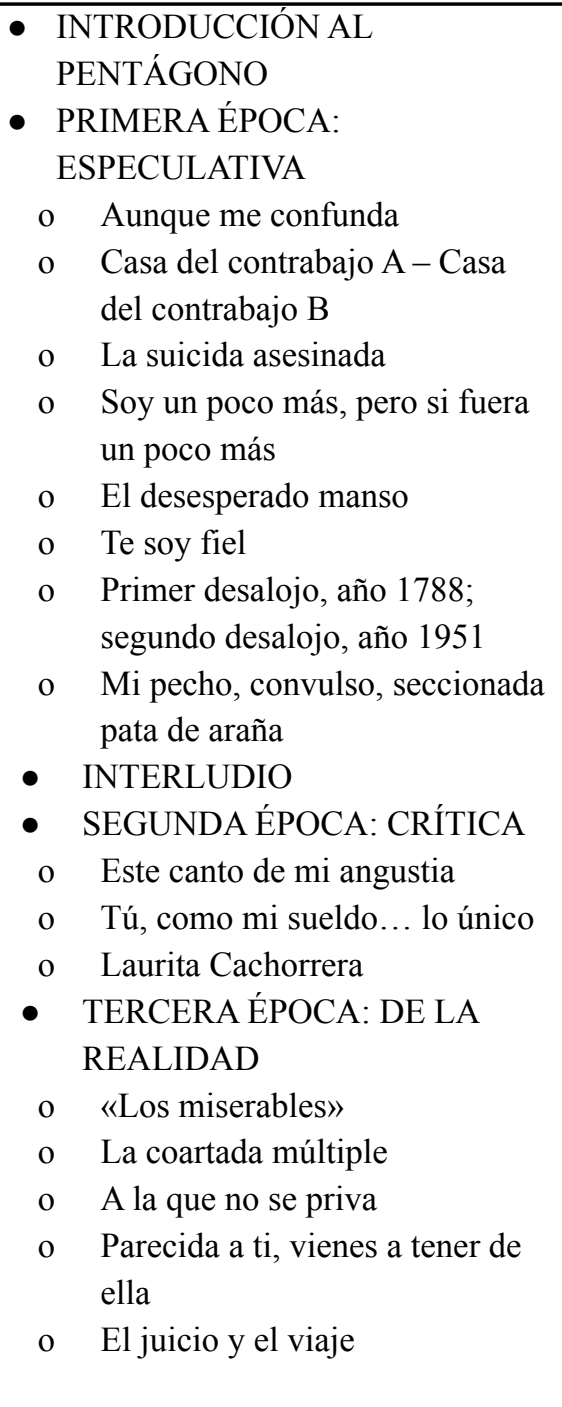 & 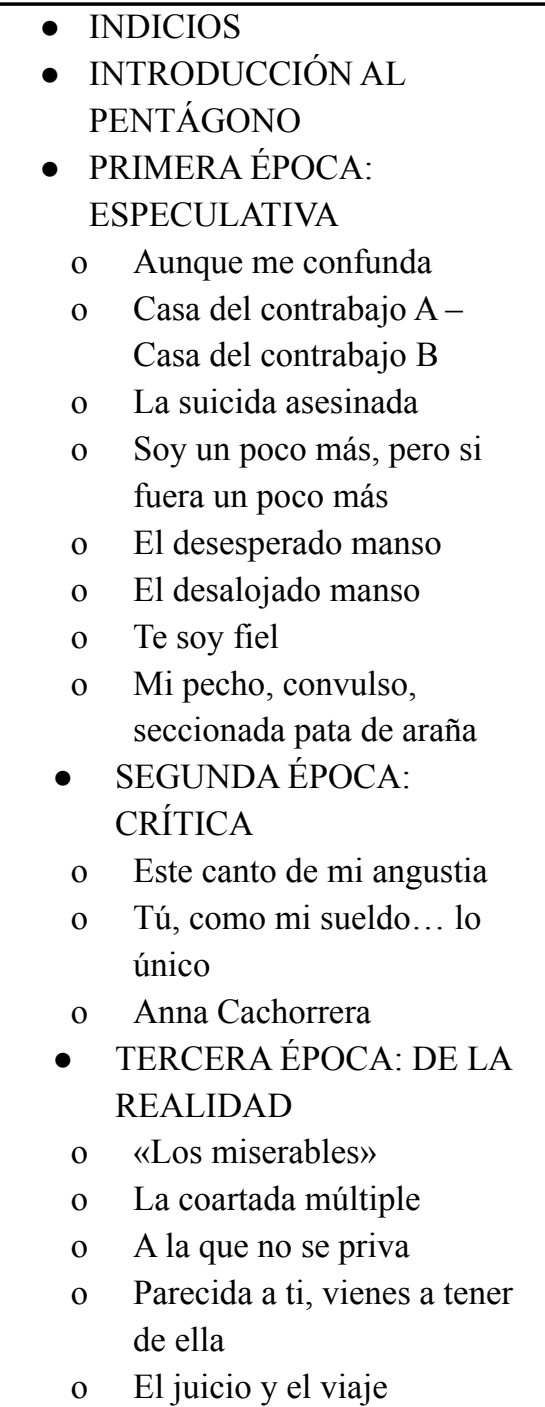 \\
\hline
\end{tabular}

\section{VARIANTES DE REESCRITURA}

*Según orden de aparición el texto:

\begin{tabular}{|l|l|l|l|l|l|l|}
\hline $\begin{array}{l}\text { Reedición de Adriana } \\
\text { Hidalgo 2018 (fiel al texto } \\
\text { de 1955) }\end{array}$ & Pág. & $\begin{array}{l}\text { Edición de 1974 por } \\
\text { Ediciones Orion }\end{array}$ & Pág. & Procedimiento \\
\hline
\end{tabular}




\begin{tabular}{|c|c|c|c|c|c|}
\hline$[1]$ & $\begin{array}{l}\text { Segunda sección del } \\
\text { primer relato: Aunque me } \\
\text { confunda iniciado con «En } \\
\text { fin, todo es culpa de éstos. } \\
\mathrm{O} \text { de esto. Porque esta } \\
\text { oficina nos pone así». }\end{array}$ & 40 & $\begin{array}{l}\text { La segunda sección del } \\
\text { primer relato (una página } \\
\text { aproximadamente) es } \\
\text { modificada por completo, } \\
\text { conservando un sentido } \\
\text { similar (descripción de la } \\
\text { oficina donde el } \\
\text { protagonista trabaja), pero } \\
\text { abandonando por completo } \\
\text { el registro coloquial en } \\
\text { virtud de un lenguaje de } \\
\text { corte más poético: "Arduo } \\
\text { va a ser distinguir si es } \\
\text { mera fantasía del } \\
\text { entresueño, cuando nace el } \\
\text { día bajo la amenaza del } \\
\text { despertador, o son fugas } \\
\text { somnolientas } \\
\text { oficinista». }\end{array}$ & 30 & Sustitución \\
\hline$[2]$ & $\begin{array}{l}\text { Penúltima sección del } \\
\text { primer relato: Aunque me } \\
\text { confunda iniciado con: } \\
\text { «Sin embargo, no siempre } \\
\text { he pensado así. Ni pienso } \\
\text { así sólo desde que él dijo } \\
\text { que volaba o que había } \\
\text { soñado que volaba o dijo } \\
\text { que yo podía volar». }\end{array}$ & 42 & $\begin{array}{l}\text { La penúltima y la última } \\
\text { sección se funden en una } \\
\text { más breve. Los dos } \\
\text { primeros párrafos son } \\
\text { suprimidos en favor de uno } \\
\text { corto donde se introduce la } \\
\text { idea del suicidio, no tan } \\
\text { literalmente presente en la } \\
\text { versión anterior: } \\
\text { «Considero la ventana. Si } \\
\text { tomase una silla y por ella } \\
\text { ascendiese al alféizar, el } \\
\text { paso siguiente, ¿me } \\
\text { precipitaría hacia la } \\
\text { profundidad o me lanzaría } \\
\text { a las alturas?» }\end{array}$ & 32 & $\begin{array}{ll}\text { Sustitución } & \text { y } \\
\text { supresión } & \end{array}$ \\
\hline$[3]$ & $\begin{array}{l}\text { El relato Casa del } \\
\text { contrabajo A. Casa del } \\
\text { contrabajo B comienza con } \\
\text { un epígrafe que reza: «Una } \\
\text { antigua historia puede } \\
\text { contarse de otra manera» }\end{array}$ & 47 & El epígrafe es suprimido & 37 & Supresión \\
\hline$[4]$ & $\begin{array}{l}\text { Relato Casa del contrabajo } \\
\text { A. Casa del contrabajo B, } \\
\text { últimarárón. } \\
\text { Conversación final entre } \\
\text { Santiago y Laura: } \\
\text { «-Amo el contrabajo, y } \\
\text { con él no se puede vivir en } \\
\text { otra parte. }\end{array}$ & 50 & $\begin{array}{l}\text { Algunos cambios } \\
\text { significativos en esta } \\
\text { sección, particularmente la } \\
\text { conversación final entre el } \\
\text { protagonista, Santiago, y } \\
\text { Anabella (antes Laura): }\end{array}$ & 40 & Sustitución \\
\hline
\end{tabular}




\begin{tabular}{|c|c|c|c|c|c|c|}
\hline & $\begin{array}{l}\text {-Tú amas la } \\
\text { promiscuidad en el } \\
\text { contrabajo. } \\
\text { Calló un momento y luego, } \\
\text { muy mansa, muy buena y } \\
\text { convincente, me explicó: } \\
\text {-No, Santiago. Te } \\
\text { equivocas. Rolando habita } \\
\text { el contrabajo conmigo } \\
\text { porque Rolando también } \\
\text { ama el contrabajo». }\end{array}$ & & $\begin{array}{l}\text { «-Pero } \frac{2}{2} \text { Santiago... } \\
\text { — quiso hacer valer-. Yo } \\
\text { amo mi instrumento. } \\
\text { — iAmas la promiscuidad } \\
\text { en el contrabajo! } \\
\text { Me miró con dulzura y } \\
\text { serenidad, como para } \\
\text { deponer mi enojo y } \\
\text { conseguir } \\
\text { entendiera: } \\
\text {-No, Santiago. } \\
\text { equivocas... } \\
\text { - iY entonces qué haces } \\
\text { con él, apretados dentro de } \\
\text { un vulgar contrabajo?... } \\
\text {-Música». }\end{array}$ & & & \\
\hline [5] & $\begin{array}{l}\text { Relato Primer desalojo, } \\
\text { año } 1788 ; \quad \text { segundo } \\
\text { desalojo, año } 1951\end{array}$ & 79 & $\begin{array}{l}\text { El relato pasa a llamarse } E l \\
\text { desalojado manso y } \\
\text { cambia de posición en el } \\
\text { libro a continuación de } E l \\
\text { desesperado manso. } \\
\text { Además, se agrega en la } \\
\text { última sección un párrafo: } \\
\text { «Dos veces me había } \\
\text { desalojado ya: la primera } \\
\text { de mi lecho matrimonial; } \\
\text { la segunda, del hogar de } \\
\text { paz en que me resigné a no } \\
\text { morir» y una frase al final: } \\
\text { «Salí murmurando contra } \\
\text { la desventaja de que } \\
\text { también haya otros que } \\
\text { sean inmortales». }\end{array}$ & 67 & $\begin{array}{l}\text { Sustitución } \\
\text { adición }\end{array}$ & \\
\hline [6] & $\begin{array}{l}\text { Breve relato Mi pecho, } \\
\text { convulso, seccionada pata } \\
\text { de araña en tres secciones } \\
\text { separadas por un asterisco } \\
\text { cada una, al igual que en } \\
\text { todos los relatos del libro. }\end{array}$ & $87-90$ & $\begin{array}{l}\text { Se divide el relato en dos } \\
\text { secciones marcadas por I y } \\
\text { II. La sección I } \\
\text { corresponde a la primera } \\
\text { sección presente en El } \\
\text { pentágono y la II a las } \\
\text { otras dos, que continúan } \\
\text { divididas por un asterisco } \\
\text { como en el resto del libro. } \\
\text { Hay significativas } \\
\text { sustituciones y adiciones } \\
\text { en la primera sección } \\
\text { respecto de la versión de } E l \\
\text { pentágono, } \\
\text { fundamentalmente en un }\end{array}$ & $83-87$ & $\begin{array}{l}\text { Sustitución, } \\
\text { supresión } \\
\text { adición }\end{array}$ & \\
\hline
\end{tabular}




\begin{tabular}{|c|c|c|c|c|c|}
\hline & & & $\begin{array}{l}\text { pasaje donde el } \\
\text { protagonista narrador se } \\
\text { compara a un vendedor de } \\
\text { zanahorias que no vende. } \\
\text { Por motivos de extensión } \\
\text { no se transcribirán, debido } \\
\text { también a que no } \\
\text { parecieran operar un } \\
\text { cambio de sentido en el } \\
\text { pasaje, sino más bien, de } \\
\text { estilo. }\end{array}$ & & \\
\hline [7] & $\begin{array}{l}\text { Parte breve, denominada } \\
\text { INTERLUDIO, solo } \\
\text { incluye un pasaje que dice: } \\
\text { «Evadido de mi lecho de } \\
\text { enfermo, ganoso de sol, de } \\
\text { un día de sol... } \\
\text { Intermedio } \\
\text { Intermedio } \\
\text { primavera-invierno, } \\
\text { parque-ciudad. Túnica de } \\
\text { sol. El aire, un aire limpio } \\
\text { y débil, está más fuerte que } \\
\text { yo. } \\
\text { Si esa muchacha me mira, } \\
\text { la tierra tendrá perfume. Si } \\
\text { no hubiera tanta gente, yo } \\
\text { cantaría, llorando. Si ella } \\
\text { estuviera aquí... Si ella } \\
\text { estuviera conmigo...» }\end{array}$ & $92-93$ & $\begin{array}{l}\text { El INTERLUDIO entre } \\
\text { PRIMERA ÉPOCA y } \\
\text { SEGUNDA ÉPOCA es } \\
\text { totalmente suprimido. }\end{array}$ & & Supresión \\
\hline [8] & $\begin{array}{l}\text { Relato titulado Laurita } \\
\text { cachorrera }\end{array}$ & 107 & $\begin{array}{l}\text { Cambia el nombre del } \\
\text { relato a Anna cachorrera, } \\
\text { consecuentemente con el } \\
\text { cambio de nombre del } \\
\text { personaje de Laura. }\end{array}$ & 101 & Sustitución \\
\hline [9] & $\begin{array}{l}\text { Último relato: El juicio y el } \\
\text { viaje, cuya primera } \\
\text { sección finaliza en: } \\
\text { «Percibí el crujido, en mi } \\
\text { pecho, que me advertía de } \\
\text { la verdad, la verdad que } \\
\text { estaba en mí mismo, y } \\
\text { comprendí porqué los } \\
\text { demás no la veían, y } \\
\text { mientras no se ve en uno } \\
\text { mismo, no se puede ver en } \\
\text { los otros a no ser por un } \\
\text { infinito acto de } \\
\text { comprensión. Pude decirlo, }\end{array}$ & 147 & $\begin{array}{l}\text { Hay una sustitución de la } \\
\text { frase final de la primera } \\
\text { sección de este relato y a } \\
\text { continuación una adición } \\
\text { significativa: «Percibí el } \\
\text { crujido, en el pecho, y } \\
\text { resolví asumir de facto, } \\
\text { aunque solo en mi interior, } \\
\text { las atribuciones judiciales, } \\
\text { y este fue el dictamen, no } \\
\text { pronunciado: 'He aquí un } \\
\text { aspecto de la sociedad } \\
\text { moralmente organizada'. }\end{array}$ & 135 & $\begin{array}{l}\text { Sustitución } \\
\text { adición }\end{array}$ \\
\hline
\end{tabular}




\begin{tabular}{|c|c|c|c|c|c|}
\hline & $\begin{array}{l}\text { confidente, humilde, } \\
\text { vencido, pero ¿quién me } \\
\text { habría escuchado? No es } \\
\text { cuestión tan solo de eso } \\
\text { llamado dignidad; es el } \\
\text { dolor, el dolor. ¡Ah, si no } \\
\text { se siente el dolor, ese } \\
\text { dolor!...». }\end{array}$ & & 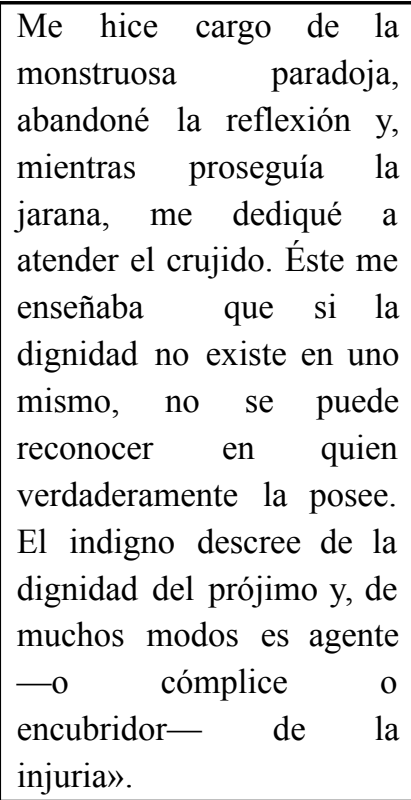 & & \\
\hline$[10]$ & $\begin{array}{l}\text { En el último relato: El } \\
\text { juicio y el viaje, hacia el } \\
\text { final, contiene la frase: «Vi } \\
\text { la capota, desde abajo; mi } \\
\text { cabeza estaba volcada } \\
\text { hacia atrás». }\end{array}$ & 151 & $\begin{array}{l}\text { La frase es modificada por: } \\
\text { «La capota se convirtió en } \\
\text { mi cielo, la veía desde } \\
\text { abajo y desde adentro: mi } \\
\text { cabeza estaba volcada } \\
\text { hacia atrás, la nuca hacía } \\
\text { apoyo en el respaldo del } \\
\text { asiento». }\end{array}$ & 147 & Sustitución \\
\hline
\end{tabular}

\section{PERSPECTIVAS CRÍTICAS A PARTIR DEL REGISTRO DE VARIANTES}

Los objetivos exploratorios de variantes en este trabajo limitan un análisis más extenso que incluya y clasifique la totalidad de las variantes no incluidas en nuestro recorte -léxicas, sintácticas, correcciones que no supongan un cambio significativo en el sentido del texto-, contribuyentes al desarrollo crítico surgido de la observación de reescritura, sobre todo en miras de tender una relación entre el proceso escritural con su contexto histórico-cultural, es decir, que se focalice en el vínculo problemático entre escritura y sociedad que indica Lois (2005a: 60). Es por ello que nos limitaremos a señalar algunas de las problemáticas y perspectivas críticas que nos ha sugerido la comparación de los materiales, considerando la posibilidad de un desarrollo superador, en un trabajo de mayores extensiones.

En «Indicios», el apartado de la edición 1974 incluído en Anabella, Di Benedetto dice: «Sobre las correcciones para la edición ha de considerarse, simplemente, que he tomado mi escrito y lo he pasado en limpio» $(1974,12)$. «Pasar en limpio», en su uso ordinario, supone tomar un texto y escribirlo nuevamente sin errores o tachaduras. ¿Qué significa «pasar en limpio» para Di Benedetto, casi veinte años después de la escritura y publicación del primer escrito? En principio, la expresión es, cuando menos, engañosa; se evidencia aquí 
que lo que modificó fue mucho más que errores sintácticos que, por otra parte, en tanto tales, debieron ser sometidos a una o más correcciones para la edición de 1955. Estamos en presencia de una reescritura del texto original, no solo por el caudal de las correcciones, sino por su importancia a nivel del significado parcial, en algunos casos, y el sentido global del texto.

Es conveniente señalar que esta tesis es contraria a la de uno de los pocos trabajos en circulación sobre estos textos: Annabella (El pentágono), de Antonio Di Benedetto: aproximaciones a una poética de la ficción de Fabiana Inés Varela. En él, Varela dice respecto de las variantes introducidas entre una edición y la otra que «responden a la búsqueda de precisión estilística y conceptual que caracteriza la prosa madura de Di Benedetto, pero no importan un cambio profundo de significado» (130). Si bien los objetivos del trabajo de Varela no son de corte genético, sino que se focaliza en la poética de la ficción implícita en la obra de Di Benedetto (128), esta afirmación es un poco apresurada en tanto que ella misma acepta, cuando menos, la importancia de una variante relacionada a la estructura y sentido del texto: la sustitución del nombre «Laura» por el de «Anabella»y, subsecuentemente, la del nombre de la obra, El pentágono por Anabella. Di Benedetto dice en «Indicios» que «Anabella» fue su primera opción para el nombre del personaje que representa el amor idealizado por el protagonista y que luego cambió por «Laura»; explica al respecto:

Anabella era una actriz del cine francés de la Edad de Oro. Estuvo en las películas "La bandera", "14 de Julio" y "Hotel del Norte". Tomé su nombre (sólo el nombre, nada biográfico le atañe) para la figura femenina central de mi obra.

Más adelante sentí la necesidad de borrarlo, tal como en el libro el protagonista, que idealiza a su amada, pretende destruirla dentro de sí mismo.

Dejé que, en lugar de Anabella, prosperara Laura, así llamada en "El Pentágono", porque este nombre correspondía a una imagen de amor y nostalgia, correspondía a una ausencia ("La Ciudad sin Laura” de Francisco Luis Bernárdez, publicado unos años antes). (1974: 11-12)

Este cambio sería menor si no fuera porque responde a un desplazamiento de foco en el sentido general de la primera obra respecto de la segunda, un corrimiento desde la poética de la ficción que Varela analiza (cuya presencia es más evidente en El pentágono) y la experimentación formal, hacia los temas, más simples quizás, del amor idealizado, el triángulo amoroso y la infracción matrimonial. Este corrimiento es justificado por Di Benedetto cuando explica que la negación original del nombre Anabella y el título de 1955 corresponden a pretensiones formales:

A expensas de este decir no, no a la sustancia del libro, se abrió paso hacia la portada una abstracción —el pentágono—, realmente no ajena a las pretensiones que yo me traía, de hacer algo distinto. (1974: 12) 
Pretensiones comprensibles atendiendo a que El pentágono es un escrito de un autor todavía joven - Di Benedetto no tiene aún treinta años cuando lo comienza- que está interesado, como ya vimos, por la experimentación formal en la novela $\longrightarrow$ o en algo similar a una novela—, en una época de auge de dicha experimentación:

Transcurría la década del $40 \mathrm{y}$, saturado de novela tradicional — sin negarla, antes bien, deslumbrado y apasionado por sus exponentes clásicos-, cometí el atrevimiento, en grado de tentativa, de "contar de otra manera". Por lo cual provoqué esta "novela en forma de cuentos". (1974: 12)

Para la edición de 1974, Di Benedetto ya estaba al tanto de algunas iniciativas de experimentación novelística que sucedieron en paralelo a la escritura de El pentágono, así como de repercusiones posteriores de la crítica respecto de este y otros textos suyos.:

Vine a saberlo hacia 1960, y mejor me enteré después, cuando algunas voces de la crítica argentina, también europea, se acordaron de "El Pentágono", "El Abandono y la Pasividad", "Declinación y Ángel” y otras imperfecciones mías, en sus nóminas y referencias sobre el Objetivismo u Objetismo, la Novela Nueva y los Precursores; en las del Absurdo por "Mundo Animal", "Caballo en el Salitral” y otra vez "El Pentágono". (1974: 12-13)

En la reescritura para la edición de 1974, frente a estos sucesos en el campo literario, los dichos de la crítica respecto de El pentágono y un Di Benedetto ya maduro y consagrado, la tentativa de experimentación formal pierde relevancia. La sustitución del nombre de la obra y la del personaje de Laura por Anabella son marcas que dan cuenta de este corrimiento desde el «contar de otra manera» y la poética de la ficción, hacia un tema más simple, propio de la historia y no de su construcción que Di Benedetto, además, señala claramente en «Indicios»: «Este libro trata de la infracción. Su anécdota particulariza la infracción matrimonial, en la atmósfera de un modo de amor, un modo curioso o absurdo» (1974: 13). La variante [4], donde hay una modificación notable del final de un relato, muestra también la acentuación del tema del triángulo en Anabella y un esfuerzo por volver el foco hacia la historia. Néspolo dice que «El Pentágono puede sumarse a la — para algunos — 'irritante' lista de una serie de textos en los que la palabra es su misma condición de existencia, puesto que la única realidad invocada es la de la escritura...». Después de haber publicado su «trilogía» (Saer, 2016: 5) —Zama, El silenciero y Los suicidas—, la escritura de Di Benedetto había pasado ya de su periodo experimental y conseguido la solidez de estilo que lo caracteriza — una «unidad estilística y temática» según Saer (2016: 5)—, por lo que las tentativas de hacer literatura a nivel del discurso ya no son necesarias: la reescritura de 1974 ensaya un texto más potable para el lector, que invoque una realidad fuera del puro procedimiento.

Si bien la corrección de estilo es notable, como evidencia la reescritura completa de un pasaje en la variante [1], en su conjunto las variantes señalan un cambio en el sentido, 
contrario a lo que dice Varela y a lo que da a entender el mismo autor con «pasar en limpio». El desplazamiento del sentido general del texto, de la dimensión discursiva del texto a la historia, es explicable en los sucesos en el campo literario de los que Di Benedetto confesamente estaba al tanto, así como en la evolución de su propia obra que también pone en juego en la nueva escritura. Ejemplo claro de esto último es la introducción de la idea de suicidio en la variante [2], para nada menor teniendo en cuenta que en 1969 Di Benedetto publica Los Suicidas, tras haber atravesado un proceso de investigación y recopilación de fuentes respecto al tema, como el carácter intertextual de esa novela demuestra: se suprimen los juegos metafóricos y el tema del suicidio se vuelve literal.

Algunos otros cambios de estructura, como la supresión del interludio en la variante [7], un texto de registro por demás poético y algo fuera de lugar, así como los cambios de nombre, posición y organización de los relatos en [5] y [6], parecieran indicar la búsqueda de una simplificación estructural y mayor unidad de la obra, posiblemente también relacionadas al corrimiento desde lo formal a lo narrativo antes señalado. En [5], el cambio de posición y de nombre del relato responde a la tendencia de otorgarle a la obra el formato de una novela más que de un libro de cuentos, ya que el nuevo nombre, «El desalojado manso», lo relaciona directamente al «El desesperado manso», el relato inmediatamente anterior en la nueva posición, indicando continuidad estructural. En este sentido, Anabella es más una novela que El pentágono.

En [6], la corrección de estilo indica, como en [1] y la supresión de [7], un intento de registro más coloquial y llano, menos poético, que atraviesa toda la reescritura de 1974. Modificación de estilo que también responde a la búsqueda de unidad estructural señalada en [5]. Sustituciones mínimas como [8], donde el cambio del nombre del personaje lleva a cambiar el título del relato, también indican que se volvió a pensar la obra como un todo con la consecuente necesidad de una nueva unidad y por el efecto encadenado que producen cambios mayores como el del nombre de la protagonista.

A nivel de los personajes, especialmente en el narrador-protagonista, es probable que en la reescritura se haya apostado por una mayor profundidad psicológica. En la variante [9], hacia el final, se incluye una cavilación del protagonista en torno a la dignidad, cuestión que en El pentágono estaba apenas sugerida. Esta profundización es constatable en la variante [2], con la inclusión más literal de la idea y la duda acerca del suicidio en los pensamientos del protagonista; en la variante [4] con la complejización del diálogo entre el protagonista y Anabella; y en [6] cuyas adiciones dan cuenta de una mayor densidad de las relaciones entre personajes. Esta densificación de la realidad de los personajes, demuestra también un vuelco hacia elementos fuera de lo meramente escritural y una unificación de la totalidad del texto en tanto novela. Por otro lado, el caso particular de [6] requeriría un análisis más profundo porque, más que una variante, responde a un conjunto de variantes relacionadas, y comporta un cambio de estructura al interior del relato que no se da de la misma manera en otros. Nos interesa mencionar este último caso, como posibilidad de microanálisis que, por lo pronto, excede a lo que aquí nos propusimos demostrar: el corrimiento del sentido general del texto 
hacia una dimensión no únicamente discursiva, no excesivamente situada en la experimentación lingüística.

\section{ALGUNAS CONCLUSIONES}

Grésillon, respecto a lo denominado por Lebrave como «la doble locución genética», dice:

... se dice comúnmente de todo escritor que se relee, lo que parece significar dos cosas: por una parte, que el escritor escribe y lee prácticamente al mismo tiempo; por otra parte que, al término de ciertos periodos de redacción, se transforma en lector crítico que vuelve a ver su escrito, de alguna manera, con los ojos de otro. Este hecho destacable, de que un mismo sujeto sea escritor y lector a la vez [...] produce, por supuesto, un tipo específico de reescritura. (2008: 169)

Retomar una escritura veinte años después supone, cuando menos, encontrarse con la extrañeza de uno mismo, ver la propia producción como la de otro. Como las lecturas que vamos acumulando modifican la manera en que leemos, la lectura de lo que se dijo de nuestros propios textos no puede sino modificar el modo en que interpretamos y valoramos nuestra propia escritura. Di Benedetto lector conocía ya el Nouveau Roman y el Objetivismo, estaba atravesado por lo que la crítica había dicho sobre su propia obra y no era más el desconocido autor del interior que fue en 1955. Como escritor, su misma producción había mejorado en cantidad y calidad: ya habían sido publicadas las tres novelas de la llamada «trilogía de la espera», su conciencia de la novela y su capacidad para escribirla habían crecido notablemente. Una reescritura es, en estas condiciones, pasado tanto tiempo, también una recontextualización. Esto significa que los sentidos del texto original no pueden permanecer inalterados tan fácilmente, están atravesados por demasiadas variables histórico-culturales, así como personales que van a tener un impacto en las nuevas versiones del texto. Las variantes existentes entre El pentágono y Anabella dan cuenta de esta recontextualización y vuelven lícito afirmar que los sentidos del texto han sido desplazados por una escritura otra; a la manera de Foucault, se escribe para perder el rostro, la identidad del escritor nunca es la misma, no puede haber correspondencia entre las nuevas escrituras con sus primeras versiones. El lector no tiene identidad fija y el escritor es, ante todo, un lector.

Queda pendiente para un trabajo de mayor extensión y alcances el registro de variantes menores que nuestro recorte dejó afuera, así como la posibilidad de un microanálisis que muestre la función específica de cada una de ellas. 


\section{BIBLIOGRAFÍA}

Foucault, Michel (2008): La arqueología del saber, Buenos Aires, Siglo XXI.

Filer, Malva E. (1982): La novela y el diálogo de los textos, México D.F., Editorial Oasis.

Grésillon, Almuth (2005): «Glosario de crítica genética», trad. Aurore Baltasar en Fernando Colla (coord.), Archivos. Cómo editar la literatura latinoamericana del siglo XX, Poitiers, Centre de Recherches Latino-Américaines, Université de Poitiers, pp. 289-297.

-(2008): «Los manuscritos literarios: el texto en todos sus estados» en Grésillon et al. Genética textual, Madrid, Arco Libros, pp. 153-179.

Lois, Élida (2005a): «De la filología a la genética textual: historia de los conceptos y de las prácticas» en Fernando Colla (coord.), Archivos. Cómo editar la literatura latinoamericana del siglo XX, Poitiers, Centre de Recherches Latino-Américaines, Université de Poitiers, pp. 47-83.

- (2005b): «Las técnicas filológicas y las innovaciones técnicas de la genética textual» en Fernando Colla (coord.), Archivos. Cómo editar la literatura latinoamericana del siglo $X X$, Poitiers, Centre de Recherches Latino-Américaines, Université de Poitiers, pp. 127-138.

Néspolo, Jimena (2004): Ejercicios de pudor, Buenos Aires, Adriana Hidalgo.

- (2018): «Lecturas impertinentes», prólogo y notas en Antonio Di Benedetto (2018a), El pentágono, Buenos Aires, Adriana Hidalgo.

Premat, Julio (2004): «Un pentágono triangular. Orígenes de la narrativa de Antonio Di Benedetto» en Río de la Plata, 26-27, Los años sesenta en el Río de la Plata, pp. 295-302.

-(2017): «Así se nace, Vanguardia, estilo, extrañeza en Di Benedetto» en Liliana Reales (ed.), Homenaje a Antonio Di Benedetto, Mendoza, Universidad de Cuyo, pp. 11-28.

Saer, Juan José (2016): «Prólogo a El silenciero» en Antonio Di Benedetto (2016a), El silenciero, Buenos Aires, Adriana Hidalgo.

Varela, Fabiana Inés (2004): «Annabella (El pentágono), de Antonio Di Benedetto: aproximaciones a una poética de la ficción», Piedra y Canto. Cuadernos del CELIM, 9-10, pp. 127-140. 


\section{BIBLIOGRAFÍA DE ANTONIO DI BENEDETTO}

Di Benedetto, Antonio (1974): Anabella, Buenos Aires, Ediciones Orión.

- (2008): Sombras, nada más..., Buenos Aires, Adriana Hidalgo.

-(2014): Zama, Buenos Aires, Adriana Hidalgo.

-(2015): Cuentos completos, Buenos Aires, Adriana Hidalgo.

- (2016a): El silenciero, Buenos Aires, Adriana Hidalgo.

- (2016b): Los suicidas, Buenos Aires, Adriana Hidalgo.

-(2016c): Escritos periodísticos, Buenos Aires, Adriana Hidalgo.

-(2018a): El pentágono, Buenos Aires, Adriana Hidalgo.

-(2018b): Prefiero la noche, prefiero el silencio, Buenos Aires, Adriana Hidalgo. 